\title{
Effects of early vs late lesions on cognitive-affective behavior in rats: $\mathrm{VMH}^{\prime}$
}

\author{
PHILLIP C. GREEN \\ UNIVERSITY OF CINCINNATI
}

Ventromedial hypothalamic lesions were produced in prepuberal and postpuberal rats to test for subsequent effects on tests of emotionality, and active and passive avoidance. Data indicated heightened emotionality, higher activity, disinhibition of PAR, but no difference in acquisition of AAR by the postpuberal operates, while failure of prepuberals to show these changes provided evidence that age at lesioning is an important variable in this area.

Recent years have seen a rapid increase in studies concerned with effects of limbic lesions on learning and emotion. McCleary (1961) found that bilateral cingulectomy in adult cats produced defects in active avoidance (AAR) while subcallosal lesions caused similar deficits in passive avoidance (PAR). The finding of PAR deficits has since been confirmed and extended to the hypothalamus by Kaada, Rasmussen, \& Kviem(1962) in the rat. Recently, however, McNew \& Thompson (1966) have demonstrated that rats sustaining lesions of various structures which include medial hypothalamus show deficiencies in both types of avoidance. Since neuroanatomical evidence (Kaada, 1960) indicates that inhibitory control from subcallosal cortex is mediated, at least partly, by ventromedial hypothalamus (VMH), this structure was selected for further investigation in the present study. In addition, another variable-the effect of age of lesion on these behaviors-concerned us, since experiments (Kling, 1962; Green \& Kling, 1966) have indicated that early lesions in cat and monkey fail to produce the changes seen in later-lesioned animals. The purpose of this research was to study the effect of VMH lesions in prepuberal (PRP) and postpuberal (PSP) rats on several tests of cognitive-affective response.

\section{Procedure}

Ss used in this study were 46 hooded rats. The two age groups were balanced for sex composition and then randomly divided into $\mathrm{E}$ and $\mathrm{C}$ groups. PRP Es were operated in single-stage procedures between 21 and 30 days of age; PSP Es were between 85 and 100 days old at the time of surgery. Electrolytic lesions were produced stereotaxically under Sodium Pentobarbital anesthesia using an enameled No. 26 nichrome wire electrode bared approximately $1 \mathrm{~mm}$ from the tip. Half of the Cs, randomly selected, received sham operations. All Ss were subsequently tested at about the same age, following which Es were sacrificed and their brains processed for histological analysis. Lesions were found to be comparable in size and locus for all operates although in several cases some ventral hypothalamus was removed with VMH.

\section{Experiment 1. Open-field test.}

In all tests the PSP group contained $10 \mathrm{Es}$ and $10 \mathrm{Cs}$, while group PRP included $11 \mathrm{Es}$ and $15 \mathrm{Cs}$. Apparatus consisted of a rectangular enclosure, 42 in. on each side, with the floor divided into squares. For half of this test an opaque startbox (SB), containing a release door, was located on a corner square. An overhead light provided illumination while a barrier prevented escape from the field. To start a trial, $S$ was retained in SB for 1 min., the door was raised, and $S$ was then observed for $5 \mathrm{~min}$. Recorded were SB-exit latency, number of squares traversed, fecal boli deposited, and amount of urine on each trial. For this test only, PSP Ss were slightly older than PRP Ss at the start of testing ( $P R P=65-75$ days; PSP $=85-100$ days).

Following completion of this phase (I), Ss were run through the open-field test again except that in phase II SB was eliminated and a trial started by placing $S$ on the center square and recording the usual measures for $5 \mathrm{~min}$.

\section{Results}

The data revealed consistent differences between Es and Cs on all measures except "urination." In Phase I (Table 1), both PSP and PRP Es showed shorter SB-exit latencies, more fecal boli deposited, and more squares traversed than their respective Cs. Mean differences between $\mathrm{Es}$ and $\mathrm{Cs}$, in both age groups, were significant on "boli" and "squares traversed" $(p<.05$, Mann-Whitney $U$ test). On the SB-exit latency measure, PSP Es took significantly less time than Cs to leave SB; however, although PRP Es also left SB sooner than Cs, this difference failed to reach significance. While no age differences were found for the "urine" measure, differences ( $p \leq .05$, Mann-Whitney $U$ test) were found between the two E groups on both phases, with PSPs reaching significantly high levels on this mea sure.

Phase II recapitulated findings for Phase I on the "boli" measure. On "squares traversed," although all groups were elevated, due to gradual disinhibition following successive exposures, PSP Es exceeded both

Table 1. VMH Open Field, Phases I and II

\begin{tabular}{lccccc} 
& & \multicolumn{2}{c}{$\begin{array}{c}\text { PSP } \\
\text { (late lesioned) }\end{array}$} & \multicolumn{2}{c}{$\begin{array}{c}\text { PRP } \\
\text { (ecrly lesioned) }\end{array}$} \\
Measure & Phase & E & C & E & C \\
\hline $\bar{M}$ No.Boli & 1 & $3.12 *$ & 1.85 & 1.62 & 0.90 \\
& 11 & 1.47 & 0.93 & 1.04 & 0.58 \\
$\bar{M}$ Squares & 1 & 6.33 & 2.50 & 16.45 & 5.93 \\
Traversed & II & 24.35 & 18.52 & 16.43 & 19.65 \\
$\bar{M}$ SB-exit & 1 & 16.44 & 24.82 & 14.61 & 17.76 \\
Latency (sec.) & & & & & \\
$\bar{M}$ Urine (mg.) & 1 & 8.33 & 9.50 & 4.91 & 3.62 \\
& 11 & 6.50 & 9.00 & 3.00 & 1.75 \\
\hline
\end{tabular}

* Each cell entry represents the mean of 15 trials. 
Table 2.

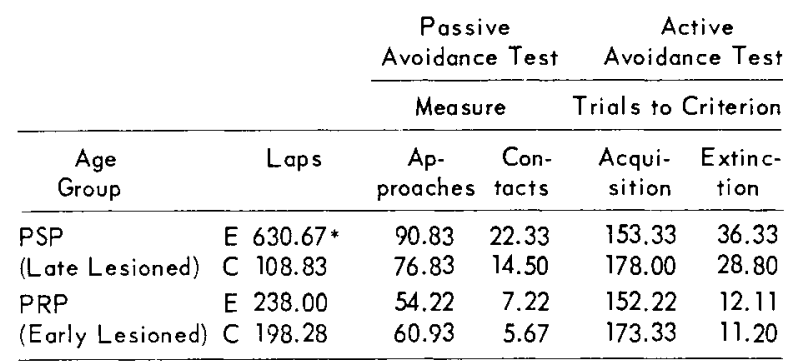

* Each cell entry represents the mean of 10 trials.

PSP Cs and PRP Es significantly $(p \leq .05)$, while no differences between PRP Es and Cs were found. A combination of Phases I and II yielded relatively the same statistical outcome of Phase II.

\section{Experiment 2. Passive avoidance.}

Passive avoidance is defined as the ability of an organism to inhibit a response which leads to punishment. Apparatus for the passive avoidance test consisted of a small compartment, 11 in. square, containing a grid floor. A small plastic water trough was centered on one wall, 2 in. above the floor. A sensing circuit, built into this unit, recorded contacts of the rat's tongue with the water, and, when required, also delivered a mild electric shock to the same organ.

All Ss initially underwent 24-hr, water deprivation. Following measurement of baseline drinking levels, ten 10-min. additional trials per $S$ were given, in which total laps, approaches to the water trough, and total trough contacts (excluding water contacts) were recorded. At the onset of testing, all Ss were approximately the same age (mean: 200 days).

\section{Results}

In this experiment results for the PSP group (Table 2) were all in the direction previously indicated in the literature for functionally related lesions. PSP Es had significantly more laps $(p<.05$, Mann-Whitney $\mathrm{U}$ test) than their Cs. Es also recorded more approaches and more contacts, although differences between PSP groups for the latter two measures fell just short of the .05 level of significance. In the PRP group, however, differences between Es and Cs lacked significance on any of the measures.

\section{Experiment 3. Active avoidance.}

In this experiment the effect of VMH lesions on active avoidance was assessed. The apparatus used in this study was a standard shuttle box, each chamber measuring 11 in. square. A 2-in.-high barrier separated the floor shock grids, each of which could be automatically activated in the proper sequence. Shock levels were initially adjusted for each $S$ and held constant thereafter. The test sequence went as follows: Habituation (1 min.) $\rightarrow$ Buzzer (CS, 4 sec.) $\rightarrow$ Shock (US). For the final second of CS, both CS and US overlapped. CS was then discontinued while US lasted a maximum of $30 \mathrm{sec}$. Ten trials per $S$ were given on each test day until criterial performance (two con- secutive days of 90 percent or better shock avoidance responses) or a maximum of 200 trials had been reached. Following acquisition, an extinction phase was run to a criterion of five consecutive CR-negative trials on two consecutive test days. At the start of the experiment, PSP and PRP rats had a mean age of 232 days. Results

Experimental Ss in both groups (Table 2) showed more rapid acquisition (fewer trials to criterion) and greater resistance (more trials to criterion) to extinction than did their respective $\mathrm{Cs}$. No significant differences were found, however, between Es and Cs in either age group on the acquisition and extinction measures.

\section{Discussion}

General findings indicated that, on the open field test, late-lesioned VMH rats (PSP) show more emotionality and explore more than related Cs. The data also revealed trends indicating that early-lesioned rats (PRP) are less emotional but more active than late-lesioned rats. On the passive avoidance test, PSP Es indicated a disinhibition of response, relative to $\mathrm{Cs}$, on "laps" by a factor of $6: 1$, and on "contacts" by a factor of $2: 1$, while PRP $E$ and $C$ mean differences showed similar trends, but these measures lacked significance. On active avoidance, neither PSP nor PRP Es showed significant defects during acquisition or extinction of this response. However, both PRP groups extinguished significantly more rapidly than PSP groups, indicating possible age effects superimposed upon the lesion. In addition to showing behavioral changes as a function of age of lesion, results of this study appear to uphold McNew and Thompson on their findings of defects on passive avoidance with medial hypothalamic lesions, but disagree with their finding of deficits in active avoidance. Additional work is still needed to further resolve the apparent lack of agreement of findings in this area.

\section{References}

Green, P. C., \& Kling, A. Effects of amygdalectomy on affective behavior in juvenile and adult macaque monkeys. APA Proc., $1966,93-94$.

Kaada, B. R. Cingulate, posterior orbital, anterior insular and temporal pole cortex. In J. Field, H. W. Magoun \& V. E. Hall (Eds.), Handbook of Physiology, 1960, Vol. 2, 1960. Pp. 13451372 .

Kaada, B. R., Rasmussen, E. W., \& Kveim, O. Impaired acquisition of passive avoidance behavior by subcallosal, septal, and hypothalamic lesions in rats. J. comp. physiol. Psychol., 1962, 55, $661-670$

Kling, A. Amygdalectomy in the kitten. Science, 1962, 137, 429430 .

McCleary, R. A. Response specificity in the behavioral effects of limbic system lesions in the cat. J. comp. physiol. Psychol., $1961,54,605-613$.

McNew, J. J., \& Thompson, R. Role of the limbic system in active and passive avoidance conditioning in the rat. J. comp. physiol. Psychol., 1966, 61, 173-180.

\section{Noie}

1. This study was supported by RD funds provided to the department of Psychology by the Graduate School of the University of Cincinnati. The author wishes to thank Mr. Richard Sadow for his assistance in this study. 